\title{
An alternative way to present a relativistic Lagrangian definition
}

\author{
M.A. De Andrade ${ }^{1}$, C. Neves ${ }^{* 10}$ \\ ${ }^{1}$ Universidade do Estado do Rio de Janeiro, Faculdade de Tecnologia, Resende, RJ, Brasil.
}

Received on February 26, 2021. Revised on June 25, 2021. Accepted on June 28, 2021.

\begin{abstract}
As long we known, the non-relativistic Lagrangian definition, $L=T-U$ with $T$ and $U$ as being, respectively, kinetic and potential energy, it is not valid at the relativistic level. This issue partially obstructs the college student's comprehension of special theory of relativity from the Lagrangian formalism. Further, it is well known that the space-time world demands essentials principles of special theory of relativity: four-vectors and Lorentz invariance. In this work, we departure from these basics concepts of space-time world in order to get a relativistic Lagrangian definition parallel to the non-relativistic one and, as a consequence, this can help the college student to better understand special theory of relativity 1 .
\end{abstract}

Keywords: Special Relativity, Lorentz Invariance, Four-vector in space-time, Lagrangian and Hamiltonian formalism.

\section{Introduction}

It is well known that the Lagrangian formalism, as well as, Hamiltonian one are important to describe classical systems, because these formalisms reveal many important features, symmetries [2, 3] for example, and allow to quantize them through some quantization processes: canonical [4] and path integral [5] quantization methods for instance. However, when it is considered relativistic systems, the usual non-relativistic Lagrangian definition ( $L=T-U)$ is not extended to the relativistic one, as the Hamiltonian formalism is, i.e., $H=T+U$. It happens because, unlike Hamiltonian, Lagrangian is defined in the configuration space. This issue embraces some concepts that are difficulty to the college student to understand. Among others things, this is a point that we want to clarify in this work.

The relativistic Lagrangian formalism is usually presented, through fundamental books in college, in an opaque way due to some assumptions considered and it is not possible to do a parallel between non-relativistic and relativistic Lagrangian definitions. Precisely, the non-relativistic Lagrangian definition, $L=T-U$, is not valid at the relativistic level. Consequently, this obliterates the student to better comprehend the special theory of relativity and, indeed, some gaps arise in the student comprehension of the special theory of relativity in the Lagrangian framework. In order to fill this gap and to promote a better way to teach special theory of relativity from the Lagrangian formalism, at college level, we start to discuss the concepts and importance of four-vector and Lorentz invariance. After that, we show

\footnotetext{
${ }^{*}$ Correspondence email address: clifford@fat.uerj.com
}

how these concepts help to formulate the relativistic Lagrangian in a straightforward and transparent way in order to facilitate the college student to understand special theory of relativity from the Lagrangian point of view.

In order to get a self-contained work, it is organized as follows: in section 2 the basics of four-vectors and the importance of Lorentz invariance are reviewed. After that, departing from this review, the relativistic Lagrangian of the free particle is obtained in a very clean and straightforward way. In section 3 , the steps that led us to obtain the relativistic Lagrangian of the free particle given in the end of the section 2 are extended to explore the dynamics of a generic interacting system. In section 4 it is considered a particle in a background electromagnetic field in order to illustrate how this previously alternative relativistic Lagrangian formulation is more effective than the usual one. Consequently, it is possible to explore a parallel between the relativistic and non-relativistic Lagrangian definitions, indeed, it is obtained a relativistic Lagrangian definition parallel to $L=T-U$. At the end, some conclusions and discussions are presented.

\section{Basics on Four-Vectors of the Special Relativity}

We start this Section describing the notation used here. The Greek indices indicate the four-dimensional spacetime with the coordinates $x^{\mu}=\left(x^{0}, x^{i}\right)$ while the Latin indices indicate the standard three-dimensional space with $i=1,2,3$. So the $x^{0}$ and $x^{i}$ refer to the timelike and the spacelike components, respectively. 
We would like to do a brief comment about the metric definition. Consider a $N$-dimensional space $\left(V^{N}\right)$ with no special structure in which tensors play a role are metric spaces, i.e., they possess a rule which assigns "distances" to pairs of neighboring points. In particular, one calls a space (pseudo-)Riemannian if there exists an invariant quadratic differential form, namely,

$$
d s^{2}=g_{a b} d x^{a} d x^{b}
$$

with $a, b=1,2, \ldots, N$ and the $g$ 's are generally functions of position and are subject only to the following restriction: $\operatorname{det}\left(g_{a b}\right) \neq 0$. As it is required that $d s^{2}$ is invariant, it follows that $g_{a b}$ must be a tensor, which is called the metric tensor, while Eq. (1) is the metric. In pseudo-Riemannian spaces such as Minkowski space, the square of a vector, given in Eq. (1), can be a positive or a negative real number and Eq. (1) is rewritten as

$$
d s^{2}=\eta_{\mu \nu} d x^{\mu} d x^{\nu}
$$

with the metric tensor $\eta_{\mu \nu}$, that reflects the nature of the space-time, as well as its inverse $\eta^{\mu \nu}$, reads as

$$
\eta_{\mu \nu}=\eta^{\mu \nu}=\text { diagonal }(+,-,-,-),
$$

and, consequently, it is get the following identity,

$$
\eta^{\mu \nu} \eta_{\nu \lambda}=\delta_{\lambda}^{\mu}
$$

with $\delta_{\lambda}^{\mu}$ as the Kronecker delta.

The transformation law of a contravariant tensor, in particular a component four-differential $d x^{\rho}$, from one coordinate system $\left\{x^{\mu}\right\}$ to another, $\left\{x^{\prime \mu}\right\}$, is expressed by

$$
d x^{\prime \mu}=\Lambda_{\rho}^{\mu} d x^{\rho},
$$

where, by the chain rule of differentiation, we have

$$
\Lambda_{\rho}^{\mu}=\frac{\partial x^{\prime \mu}}{\partial x^{\rho}} .
$$

By using, again, the chain rule of differentiation, we can take the following trivial identity

$$
\Lambda_{\rho}^{\mu}\left(\Lambda^{-1}\right)_{\nu}^{\rho}=\frac{\partial x^{\prime \mu}}{\partial x^{\rho}} \frac{\partial x^{\rho}}{\partial x^{\prime \nu}}=\frac{\partial x^{\prime \mu}}{\partial x^{\prime \nu}}=\delta_{\nu}^{\mu} .
$$

From which, we can read

$$
\frac{\partial x^{\rho}}{\partial x^{\prime \nu}}=\left(\Lambda^{-1}\right)_{\nu}^{\rho}
$$

After the contraction of both sides of the Eq. (8) with the four-derivative operator $\frac{\partial}{\partial x^{\rho}}$, we get the transformation law of the four-derivative operator as being

$$
\frac{\partial}{\partial x^{\prime \nu}}=\left(\Lambda^{-1}\right)^{\rho}{ }_{\nu} \frac{\partial}{\partial x^{\rho}} \quad \text { or } \quad \partial_{\nu}^{\prime}=\left(\Lambda^{-1 T}\right)_{\nu}^{\rho} \partial_{\rho},
$$

where the superscript $T$ means the transpose operation.
Whenever the motion of the particle is taken in consideration, space-time comes into play, then we must use the metric tensor $\eta_{\mu \nu}$. With the intervention of the metric tensor, an additional condition for $\Lambda_{\nu}^{\mu}$, or for the corresponding transformation matrix $\Lambda$, can be found in a way to provide invariant scalar forms in four-dimensional space-time, like the distance in the three-dimensional space. With the aim to simplify the procedure, we can employ the usual notation in which the space-time metric tensor $\left(\eta_{\mu \nu}\right)$ and its inverse $\left(\eta^{\mu \nu}\right)$ can be used to lower or raise indices. Thus, using the inverse of the metric tensor to write the four-differentials on both sides of the Eq. (5) with their indices lowered, as well as in $d x^{\prime \mu}=\eta^{\mu \alpha} d x_{\alpha}^{\prime}$, it can be verified that

$$
d x_{\nu}^{\prime}=\left(\eta \Lambda \eta^{-1}\right)_{\nu}^{\rho} d x_{\rho} .
$$

So that $d x_{\nu}^{\prime}$ is covariant four-differential, whose contraction with its contravariant partner $d x^{\prime \nu}$ results in an invariant scalar quantity, the transformation matrix in Eq. 10 must be the same as that found for the fourderivative operator in Eq. (9), therefore

$$
\eta \Lambda \eta^{-1}=\Lambda^{-1 T} \quad \text { or } \quad \Lambda^{T} \eta \Lambda=\eta .
$$

The transformation of the four-differential performed by the $\Lambda_{\nu}^{\mu}$, which satisfies the Eq. (11), is called a Lorentz transformation ${ }^{1}$ Therefore, it assures the invariance of the quadratic form, i.e., the invariance of the metric, namely:

$$
d x^{\prime \mu} d x_{\mu}^{\prime}=d x^{\mu} d x_{\mu}=\text { invariant. }
$$

A generic contravariant four-vector must have the transformation matrix satisfying the Eq. (11) and its transformation law, as that of the four-differential in Eq. (5), then

$$
A^{\prime \mu}=\Lambda_{\nu}^{\mu} A^{\nu} .
$$

A generic covariant four-vector must have the transformation matrix satisfying the Eq. (11) and its transformation law, as that of the four-derivative in Eq. (9),

$$
B_{\mu}^{\prime}=\left(\Lambda^{-1}\right)_{\mu}^{\rho} B_{\rho}
$$

In such a way that the contraction of a contravariant four-vector with other covariant one, and vice-versa, results in a Lorentz invariant scalar:

$$
A^{\prime \mu} B_{\mu}^{\prime}=A^{\nu} B_{\nu}
$$

Any four-vector can be split into timelike and spacelike components and the metric tensor can be used to lower the index as

$$
A^{\mu}=\left(A^{0}, \boldsymbol{A}\right) \text { and } A_{\mu}=\eta_{\mu \nu} A^{\nu}=\left(A^{0},-\boldsymbol{A}\right),
$$

\footnotetext{
${ }^{1}$ Lorentz transformation encompasses a variety of transformations like spatial rotations [3], Lorentz boosts [6], discrete symmetries transformations, let it be said that not all Lorentz-invariant laws are expressible as relations between 4-vectors and scalars; some require 4-tensors and, in quantum mechanics, spinors transformations, which is a representation of Lorentz-invariant laws [7.
} 
where $A^{0}$ is the timelike component and $\boldsymbol{A}$ is the vector assembled with the spacelike components $A^{i}$. If we go ahead and write that $A_{\mu}=\left(A_{0}, A_{i}\right)$ then $A_{0}=A^{0}$ and $A_{i}=-A^{i}$. Thus, the invariant scalar of the Eq. takes the following form

$$
A^{\prime 0} B^{\prime 0}-\boldsymbol{A}^{\prime} \cdot \boldsymbol{B}^{\prime}=A^{0} B^{0}-\boldsymbol{A} \cdot \boldsymbol{B}
$$

The four-derivative operator $\left(\partial_{\mu}\right)$ is split into timelike and spacelike components according the identity $\partial_{\nu} x^{\mu}=\delta_{\nu}^{\mu}$, which can be verified without the intervention of the metric tensor in a similar way to Eq. (7) with the interchange of $\Lambda$ and $\Lambda^{-1}$. So, for the four-derivative operator, it's worth

$$
\partial_{\mu}=\left(\partial_{0}, \nabla\right) \quad \text { and } \quad \partial^{\mu}=\eta^{\mu \nu} \partial_{\nu}=\left(\partial_{0},-\nabla\right) .
$$

Let $d x^{\mu}$ to be the infinitesimal four-displacement of the particle, which obviously behaves as a contravariant vector under Lorentz transformation. Splitting $d x^{\mu}$ into timelike and spacelike components as follows

$$
d x^{\mu}=\left(d x^{0}, d \boldsymbol{r}\right),
$$

where $d x^{0}=c d t$, with $c$ as being the speed of light in the vacuum and $d t$ as the infinitesimal time interval spent by the particle to move along the infinitesimal displacement $d \boldsymbol{r}$, whose components are $d x^{i}$. The time derivative of the four-position of the particle will be represented by $v^{\mu}$,

$$
v^{\mu}=\frac{d x^{\mu}}{d t}=(c, \boldsymbol{v})
$$

where $\boldsymbol{v}=d \boldsymbol{r} / d t$ is the ordinary velocity of the particle; $v^{\mu}$ isn't a four-vector because its contraction with its partner $v_{\mu}$ depends on $\boldsymbol{v}^{2} \equiv \boldsymbol{v} \cdot \boldsymbol{v}$, that isn't a Lorentz invariant quantity:

$$
v^{\mu} v_{\mu}=c^{2}-\boldsymbol{v}^{2}=c^{2}\left(1-\frac{\boldsymbol{v}^{2}}{c^{2}}\right)=\frac{c^{2}}{\gamma^{2}},
$$

where $\gamma$ is the Lorentz factor

$$
\gamma=\frac{1}{\sqrt{1-\frac{\boldsymbol{v}^{2}}{c^{2}}}}
$$

Obviously, a genuine four-vector with the dimension of velocity which undergoes a Lorentz transformation like a contravariant vector is $\gamma v^{\mu}$, because its contraction with its covariant partner, according to Eq. (21), yields the Lorentz invariant quantity $c^{2}$. So we can define the four-velocity $u^{\mu}$ as

$$
u^{\mu}=\gamma v^{\mu} \quad \Rightarrow \quad u^{\mu} u_{\mu}=c^{2} .
$$

Here, we would like to point out that the notation for four-velocity follows two basic text books [3, 8]. Moreover, we would to explore the relation given in Eq. (23). First, consider a particle in a reference frame $S$ with a non null arbitrary velocity $\boldsymbol{v}$, then Eq. 23) is obtained with the help of Eq. (20), Eq. (21) and Eq. (22). Now, consider another reference frame $S^{\prime}$, where this particle is momentarily at $\operatorname{rest}\left(\boldsymbol{v}^{\prime}=0\right)$. In $S^{\prime}$ the Lorentz factor is $\gamma^{\prime}=1$ and, due to Lorentz invariance of the Eq. (23), the Einstein second postulate [1, 6], $v^{0}=v^{0 \prime}=$ $c$, is justified.

The four-momentum of a free particle with rest mass $m$ is defined by

$$
p^{\mu}=m u^{\mu}=\gamma m v^{\mu} .
$$

Similar to what happens to the four-velocity $u^{\mu}$, a general Lorentz boost on the four-momentum $p^{\mu}$ changes from one reference frame $S$, where the particle has an ordinary momentum $\boldsymbol{p}=\gamma \boldsymbol{m} \boldsymbol{v}$, to another $S^{\prime}$, where the particle is momentarily at rest $\boldsymbol{p}^{\prime}=\mathbf{0}$.

Thus, we have at our disposal two different four-vectors associated with the particle, the fourdisplacement $d x^{\mu}$ and the four-momentum $p^{\mu}$ that can be combined to form three Lorentz invariant scalars: (i) $d x^{\mu} d x_{\mu}$; (ii) $p^{\mu} p_{\mu}$; (iii) $p^{\mu} d x_{\mu}$.

We can assemble the first Lorentz invariant quadratic scalar by combining the four-displacement $d x^{\mu}=v^{\mu} d t$ with its covariant partner:

$$
d s^{2}=d x^{\mu} d x_{\mu}=v^{\mu} v_{\mu} d t^{2}=\frac{c^{2} d t^{2}}{\gamma^{2}},
$$

where it was used the identity given in Eq. 21). The quantity $d s$ is the infinitesimal interval [6], which can be written as

$$
d s=\frac{c d t}{\gamma}=c d t \sqrt{1-\frac{\boldsymbol{v}^{2}}{c^{2}}}=c d \tau .
$$

So, from the Lorentz invariance of $d s$, we get

$$
\frac{d t}{\gamma}=\frac{d t^{\prime}}{\gamma^{\prime}}=d \tau
$$

where $d \tau$ is the infinitesimal time interval that is the numerator of a supposed fraction whose denominator is $\gamma^{\prime \prime}=1$. Thus, this time interval was measured in the reference frame where the particle is momentarily at rest. So, $d \tau$ is called the infinitesimal proper time. As it can be understood from Eq. (27), time intervals have different values if measured in different reference frames, therefore time is a relative quantity, that is the essence of the special relativity.

After that, combining $p^{\mu}=\gamma m v^{\mu}$ with its covariant partner, using the Eq. 21, we can write the second Lorentz invariant quadratic scalar as

$$
p^{\mu} p_{\mu}=(m c)^{2}
$$

The four-momentum of the particle split into timelike and spacelike components can be read as

$$
p^{\mu}=\left(\frac{H_{\text {free }}}{c}, \boldsymbol{p}\right),
$$


where $H_{\text {free }}=\gamma m c^{2}$ is the energy of the free particle. So, we can get a Lorentz invariant quadratic scalar as

$$
p^{\mu} p_{\mu}=\frac{H_{\text {free }}^{2}}{c^{2}}-\boldsymbol{p}^{2} .
$$

Then, by comparing Eq. (28) with Eq. 30), the energymomentum relation is obtained as being

$$
\frac{H_{\text {free }}^{2}}{c^{2}}-\boldsymbol{p}^{2}=(m c)^{2} \text {. }
$$

It has already been calculated the quadratic Lorentz invariants by combining $d x^{\mu}$ and $p^{\mu}$ with their respective covariant partners. The next task will be obtain the third Lorentz invariant scalar by combining the two unique different four-vectors available for the free particle, $p^{\mu}=m u^{\mu}$ with $d x_{\mu}=u_{\mu} d \tau$. Then using the Eq. 23 , $u^{\mu} u_{\mu}=c^{2}$, and Eq. (26), expressed as $d s=c d \tau$, we obtain

$$
p^{\mu} d x_{\mu}=m c d s=-d S_{\mathrm{free}}=-L_{\mathrm{free}} d t,
$$

where $d S_{\text {free }}$ is the Lorentz invariant infinitesimal action for the free particle [6], which means that the Physics can not depend on the reference frame. The $L_{\text {free }}$ is the corresponding Lagrangian for the free particle, which by the use of $d x_{\mu}=v_{\mu} d t$ in Eq. 32, it renders to

$$
L_{\mathrm{free}}=-p^{\mu} v_{\mu} .
$$

Therefore, eliminating $p^{\mu}$ in the expression for $L_{\mathrm{free}}$ in Eq. (33) through the use of the four-momentum of the free particle given in Eq. (24), $p^{\mu}=\gamma m v^{\mu}$, then employing the Eq. 21, we get

$$
L_{\mathrm{free}}=-\frac{m c^{2}}{\gamma} .
$$

As $\gamma v^{\mu}=u^{\mu}$ and from Eq. (33), it is obtained that $\gamma L_{\text {free }}=-p^{\mu} u_{\mu}$, which is a Lorentz invariant scalar because both the four-momentum $p^{\mu}$ and four-velocity $u_{\mu}$ are genuine four-vectors, the same result can be verified from Eq. (34). So, $L_{\text {free }}$ changes with the changing of the reference frame. This result is valid for any relativistic Lagrangian, i.e., a relativistic Lagrangian never will be a Lorentz invariant scalar. On the other hand, a non-relativistic Lagrangian is always a Galilean invariant scalar, because the time interval $d t$ is a Galilean invariant scalar.

\section{Dynamics of Interacting System by Four-Vectors I}

In general, when we assemble a four-quantity in a naive way, gathering the ordinary vector of three-dimensional space in the simplest way, as an example, $v^{\mu}=\left(v^{0}, \boldsymbol{v}\right)$, where $\boldsymbol{v}$ is the ordinary three-dimensional velocity, this one obviously will not transform like a Lorentz vector. However, it can be useful for calculations, because we can always express a genuine four-vector in terms of "non-four-vectors", namely: $u^{\mu}=\gamma v^{\mu}$, where $u^{\mu}$ is an authentic four-velocity and they can evolve during calculation in a way that, sometimes, is useful or subtle, for example, as in the expression obtained in Eq. (33) for the Lagrangian of the free particle.

The technique used here is to assume that the timelike and spacelike components of $v^{\mu}$ are variable quantities and, in the end, to submit its timelike component to the Einstein's second postulate [1]: $v^{0}=c=$ constant.

In order to extend the procedure described in the previous section for an interacting system, it is considered a particle in a generic background field. This interacting system has its dynamics governed by a Lagrangian $(L)$, which must have among its terms the one that corresponds to the interaction in which the particle is subjected. Therefore, the timelike and spacelike components of the generalized four-momentum $P^{\mu}$ of an interacting system [6] are defined by the following operation ${ }^{2}$

$$
P^{0}=-\frac{\partial L}{\partial v^{0}} \quad \text { and } \quad \boldsymbol{P}=\frac{\partial L}{\partial \boldsymbol{v}} .
$$

By inspection of Eq. 35 and with $P^{\mu}=\left(P^{0}, \boldsymbol{P}\right)$ and $v_{\mu}=\left(v^{0},-\boldsymbol{v}\right)$, the generalized four-momentum of an interacting system is written as

$$
P^{\mu}=-\frac{\partial L}{\partial v_{\mu}} .
$$

With the aim to approach the dynamics of interacting system, we can start from the Legendre transformation which, in general, links the Lagrangian $L$ to the Hamiltonian $H$ [6], namely,

$$
H=\frac{\partial L}{\partial \boldsymbol{v}} \cdot \boldsymbol{v}-L
$$

From Eq. 29, it can be inferred the relation $H_{\text {free }}=$ $p^{0} c$ and, consequently, the energy-momentum relation. Then, based on covariance, in general, the Hamiltonian of the interacting system can be defined as being

$$
H=P^{0} c=P^{0} v^{0} .
$$

Therefore, from Eq. (35) and Eq. (38), the Legendre transformation in Eq. (37) can be expressed in a compact way as

$$
L=-P^{0} v^{0}+\boldsymbol{P} \cdot \boldsymbol{v},
$$

that is,

$$
L=-P^{\mu} v_{\mu}
$$

\footnotetext{
2 The symbol $\frac{\partial L}{\partial \boldsymbol{v}}$ represents the vector whose components are the derivatives of $L$ with respect to the corresponding components of $\boldsymbol{v}$ 6].
} 
Therefore, with the use of the four-vectors, the relation between the Lagrangian of the interacting system and the generalized four-momentum takes a very simple form, which resembles $L_{\text {free }}$, given in Eq. (33). The $v_{\mu}$ derivative of both sides of the Eq. 40 is

$$
\frac{\partial L}{\partial v_{\mu}}=-\left(\frac{\partial P^{\nu}}{\partial v_{\mu}}\right) v_{\nu}-P^{\mu} .
$$

Then, in the relativistic case, the compatibility between the formal definition of generalized four-momentum given in Eq. (36) and the Legendre transformation in the compact four-dimensional form given in Eq. 40 is achieved through the validity of the following constraint

$$
\left(\frac{\partial P^{\nu}}{\partial v_{\mu}}\right) v_{\nu}=0
$$

Note that Eq. (36) is restored when Eq. 42 is replaced in Eq. 41,.

On the other hand, we can departure from the compact Legendre transformation given in Eq. (40), multiplying both its sides with the infinitesimal time interval $d t$ to obtain an expression, similar to the one for the free particle given in Eq. (32), in this case for the Lorentz invariant infinitesimal action of the interacting system,

$$
d S=L d t=-P^{\mu} d x_{\mu}
$$

where $d x_{\mu}=v_{\mu} d t$ is the infinitesimal four-displacement. Note that the Lagrangian $L$ is a non-Lorentz invariant scalar, as well as the infinitesimal time interval $d t$. However the product of both provides a Lorentz invariant scalar, because $L d t=(\gamma L) d \tau$, where both $\gamma L$ and proper time interval $d \tau$ are Lorentz invariant scalars. Thus, from Eq. (43), the generalized four-momentum can be also expressed in terms of the action functional $S$ as

$$
P^{\mu}=-\frac{\partial S}{\partial x_{\mu}},
$$

whose timelike and spacelike components can be read as

$$
P^{0}=-\frac{\partial S}{\partial x^{0}} \quad \text { and } \quad \boldsymbol{P}=\nabla S
$$

which can be used together with the equation for Hamiltonian, Eq. (38), to obtain the Hamilton-Jacobi equation for an interacting system [6].

The recipe to obtain the relativistic Lagrangian $L$ through the Hamiltonian $H$ is straightforward: by knowing that $H=P^{0} c$, we can identify $P^{0}$ as a function of $v^{0}$ which, by the covariance of all the components, leads us to $P^{\mu}$ as a function of $v^{\mu}$. The Lagrangian can then be found through the expression given in Eq. 40, which can be expressed in the usual shape after the use of the identity given in Eq. (21).

\section{Dynamics of Interacting System by Four-Vectors II}

In order to illustrate the previous proposed procedure, an interacting system is assumed. Consider, as example, a particle with rest mass $(m)$ and electric charge $(e)$ in a background electromagnetic four-potential $\left(A^{\mu}\right)$ split in timelike and spacelike component as

$$
A^{\mu}=\left(A^{0}, \boldsymbol{A}\right),
$$

in which $A^{0}$ and $\boldsymbol{A}$ are, respectively, the scalar and vector potentials, whose connection with the threedimensional electric $(\boldsymbol{E})$ and magnetic $(\boldsymbol{B})$ fields are given by

$$
\boldsymbol{E}=-\nabla A^{0}-\frac{1}{c} \frac{\partial \boldsymbol{A}}{\partial t} \quad \text { and } \quad \boldsymbol{B}=\boldsymbol{\nabla} \times \boldsymbol{A},
$$

which can be explored in some basics references [3, 6] 6$]$.

The standard relativistic Hamiltonian for this system, such as the one found in Ref. [6], is expressed in analogy to the non-relativistic Hamiltonian by adding the contribution of potential energy $\left(e A^{0}\right)$ to the relativistic Hamiltonian of free particle, namely ${ }^{3}$

$$
H=\gamma m c^{2}+e A^{0}
$$

which can be interpreted as being energy only for static situations 4 i.e., electromagnetic field does not depend on the time and, consequently, the Lagrangian for the electric charge particle also does not depend explicitly on the time and the energy is the Hamiltonian given in Eq. 48.

With the intention to obtain the relativistic Lagrangian for an interacting system, we must follow the recipe described in the end of the Section 3 , for an interacting system the relativistic Hamiltonian is given by $H=P^{0} c$, thus the division of both sides of Eq. 48 by $c$ results in

$$
P^{0}=\gamma m c+\frac{e}{c} A^{0}
$$

As $P^{0}$ is the timelike component of the four-vector $P^{\mu}$ and by the covariance of all the components of $P^{\mu}$, it can be induced that

$$
P^{\mu}=\gamma m v^{\mu}+\frac{e}{c} A^{\mu}
$$

\footnotetext{
3 On the relativistic Lagrangian $L$, which must always be expressed in terms of the ordinary, time, position and velocity, we must use the standard Lorentz factor given in Eq. 22. However, for the Hamiltonian $H$ to be well defined, it must always be expressed in term of the ordinary, time, position and momentum; in this case, the alternative form of the Lorentz factor, written in terms of the momentum of a free particle $(\boldsymbol{p})$, reads as

$$
\gamma=\sqrt{1+\frac{p^{2}}{(m c)^{2}}}
$$

4 Vide chapter 3, section 19, of Ref. 6] and for a nice discussion about how Hamiltonian plays the role of energy, see chapter 2, section 2 and 3, of Ref. 8].
} 
where $P^{\mu}$ is the generalized four-momentum of the electromagnetic field. Note that the first term in the right hand size of the previous expression is the fourmomentum $p^{\mu}$ of the free particle. Inserting Eq. (50) into Eq. (40), we get the relativistic Lagrangian for the interacting particle,

$$
L=-\gamma m v^{\mu} v_{\mu}-\frac{e}{c} A^{\mu} v_{\mu} .
$$

Note that $\gamma L$ is a Lorentz invariant scalar. The Lagrangian in Eq. 51 can be simplified by using Eq. (21), which renders to

$$
L=-\frac{m c^{2}}{\gamma}+\frac{e}{c} \boldsymbol{A} \cdot \boldsymbol{v}-e A^{0}
$$

An alternative and simplest way to obtain the generalized four-momentum via Lagrangian is to express the Lagrangian with all its terms multiplied by the common factor $v_{\mu}$, as in Eq. (51), and to obtain the generalized four-momentum through the comparison with Eq. (40). For the same task, we can also employ the derivative in Eq. (36), which seems more familiar, but it can only be done considering that $v_{0}$ is a variable quantity, as it was assumed in the beginning of the Section 3. With the aim to justify the validity of these two procedures to obtain the generalized four-momentum in the scope of electromagnetism, it appears that from Eq. 50 , it is possible to get an equation similar to Eq. (32) when interacting particle is considered; if there is an electromagnetic field interacting with a particle, then

$$
P^{\mu} d x_{\mu}=m c d s+\frac{e}{c} A^{\mu} d x_{\mu}=-d S=-L d t,
$$

where $d S$ and $L$ are, respectively, the infinitesimal action and Lagrangian for the particle in the electromagnetic field [6].

Next, to confirm the equivalence of both methods to get the generalized four-momentum, one through the Eq. (36) and the other by Eq. 40, the constraint given in Eq. 42 can be verified in the case of the electromagnetic field, vide Appendix A. So, the compatibility between the Eq. (36) and Eq. (40) is consolidated. Therefore, both ways of proceeding are equally legitimate and they provide the same result for $P^{\mu}$, which is the one that is induced by covariance in Eq. 50 .

On the other hand, the kinetic energy $T$ is known as being

$$
T=H_{\text {free }}-m c^{2}=(\gamma-1) m c^{2} .
$$

In order to evidence the kinetic energy in the expression of the Lagrangian in Eq. (52), we can write it as

$$
L=-m c^{2}+\frac{(\gamma-1) m c^{2}}{\gamma}+\frac{e}{c} \boldsymbol{A} \cdot \boldsymbol{v}-e A^{0} .
$$

So, the relativistic Lagrangian of the interacting particle can be expressed in a similar way to the non-relativist one as

$$
L=-m c^{2}+\frac{T}{\gamma}-U+e \boldsymbol{A} \cdot \frac{\boldsymbol{v}}{c},
$$

where the potential energy was represented by $U=$ $e A^{0}$. In contrast with the relativistic Hamiltonian of interacting particle, given in Eq. (48), we get

$$
H=m c^{2}+T+U \text {. }
$$

In the extreme non-relativistic regime, time is an absolute quantity, as in Galilean relativity and Newtonian Mechanics. Consequently, all the Lorentz factors in the denominators of Eq. (27) must be always equal to one, independently of the velocity $(\boldsymbol{v})$. Therefore, from the Eq. 27) and Eq. 22, we get5

$$
\gamma=1, \quad c=\infty \quad \text { and } \quad T=\frac{1}{2} m \boldsymbol{v}^{2} .
$$

In this case, we must perform an operation like a "renormalization" in order to eliminate the constant infinity energy $m c^{2}$, i.e., absorbing it into the Lagrangian, Eq. (56), and Hamiltonian, Eq. (57), in such a way that the corresponding redefined non-relativistic Lagrangian and Hamiltonian can be written as 2

$$
\begin{aligned}
L & =T-U, \\
H & =T+U .
\end{aligned}
$$

We would like to emphasize that any relativistic Lagrangian can never be presented in the simple shape like that of the non-relativistic one in Eq. (59). In fact, they have very different shapes, because in the latter, we should subtract the rest energy, consider the extra factor $1 / \gamma$ on the kinetic energy and add a term that consists of a scalar product of vectors to make it equal to that of the Eq. (56). On the other hand, the relativistic Hamiltonian in Eq. (57), except for the addition of the rest energy, has its shape very similar to the non-relativistic one in Eq. 60.

\section{Conclusion}

It is well known that the Lagrangian, at the nonrelativistic level, is defined as being $L=T-U$. However, this definition is not valid at the relativistic level, indeed, it was shown in this work. According to Eq. (56), the part of the relativistic Lagrangian that corresponds to the non-relativistic one is $\frac{T}{\gamma}-U$. Due to this, it is also well known that this issue is not so clear to the college student and, consequently, the understanding of special theory of relativity from the Lagrangian formalism is jeopardized. In this work it was presented an alternative procedure, even when an

\footnotetext{
${ }^{5}$ For the kinetic energy $T$ vide Appendix $B$
} 
interacting system is considered, to teach special theory of relativity from the Lagrangian formalism based on the four-vectors and Lorentz invariance. This demonstrated, once again, the profound importance of these fundamental concepts of special theory of relativity. Here, it was explained in a detailed way the meaning and the origin of proper time, the energy-momentum relation and action; all of these three quantities are due to the four-vector framework and Lorentz invariance. In order to illustrate this procedure, it was considered the free particle and a charged particle in a background electromagnetic field. Consequently, it was possible to make a parallel between the definitions of relativistic and non-relativistic Lagrangian definitions.

\section{Acknowledgments}

C. Neves and M. A. De Andrade thank to Brazilian Research Agencies (CNPq and FAPERJ) for partial financial support. Further, we would like to thank the Reviewers for a careful reading of the manuscript and the valuable suggestions.

\section{Supplementary material}

\section{A: The four-momentum of the electromagnetic field}

Starting from the standard generalized four-momentum of the electromagnetic field

$$
P^{\nu}=\gamma m v^{\nu}+\frac{e}{c} A^{\nu}
$$

Knowing that the electromagnetic four-potential, $A^{\nu}$, depends only on the four-position, the $v_{\mu}$ derivative of the generalized four-momentum is

$$
\frac{\partial P^{\nu}}{\partial v_{\mu}}=\left(\frac{\partial \gamma}{\partial v_{\mu}}\right) m v^{\nu}+\gamma m \eta^{\mu \nu} .
$$

It follows that

$$
\left(\frac{\partial P^{\nu}}{\partial v_{\mu}}\right) v_{\nu}=\left(\frac{\partial \gamma}{\partial v_{\mu}}\right) m v^{\nu} v_{\nu}+\gamma m v^{\mu}
$$

However, from Eq. 21 it is known that $v^{\nu} v_{\nu}=\frac{c^{2}}{\gamma^{2}}$, thus

$$
\left(\frac{\partial P^{\nu}}{\partial v_{\mu}}\right) v_{\nu}=\left(\frac{\partial \gamma}{\partial v_{\mu}}\right) \frac{m c^{2}}{\gamma^{2}}+\gamma m v^{\mu}
$$

Since $\gamma=c\left(v^{\nu} v_{\nu}\right)^{-1 / 2}$, then its $v_{\mu}$ derivative can be obtained as

$$
\frac{\partial \gamma}{\partial v_{\mu}}=-c v^{\mu}\left(v^{\nu} v_{\nu}\right)^{-3 / 2}=-\gamma^{3} \frac{v^{\mu}}{c^{2}} .
$$

Using the previous result in Eq. A4,

$$
\left(\frac{\partial P^{\nu}}{\partial v_{\mu}}\right) v_{\nu}=\left(-\gamma^{3} \frac{v^{\mu}}{c^{2}}\right) \frac{m c^{2}}{\gamma^{2}}+\gamma m v^{\mu}
$$

Then, we get again, for the specific case of the electromagnetic field, the constraint of the Eq. 42,

$$
\left(\frac{\partial P^{\nu}}{\partial v_{\mu}}\right) v_{\nu}=0
$$

\section{B: The relativistic kinetic energy}

The relativistic kinetic energy can be algebraically manipulated as

$$
\begin{aligned}
T & =(\gamma-1) m c^{2} \\
& =\left(\frac{\gamma^{2}-1}{\gamma+1}\right) m c^{2} \\
& =\left(\frac{\gamma^{2}}{\gamma+1}\right)\left(\frac{\gamma^{2}-1}{\gamma^{2}}\right) m c^{2} \\
& =\left(\frac{\gamma^{2}}{\gamma+1}\right)\left(1-\frac{1}{\gamma^{2}}\right) m c^{2} \\
& =\left(\frac{\gamma^{2}}{\gamma+1}\right)\left[1-\left(1-\frac{\boldsymbol{v}^{2}}{c^{2}}\right)\right] m c^{2}, \\
T & =\left(\frac{\gamma^{2}}{\gamma+1}\right) m \boldsymbol{v}^{2} .
\end{aligned}
$$

In the extreme non-relativistic regime, when we take $\gamma=1$ and $m c^{2}=\infty$ in Eq. A8, it results in $0 \cdot \infty$, which is a value that can't be calculated. However, we can change from this indeterminate form to a determined one through the algebraic manipulation shown above. Therefore, if we take $\gamma=1$ in the equivalent Eq. (A9), we will obtain the usual expression for the non-relativistic kinetic energy.

\section{References}

[1] H.A. Lorentz, A. Einstein and H. Minkowski, The Principle of Relativity (Dover Publications, New York, 1952).

[2] L. Landau and E. Lifshitz, Mechanics (ButterworthHeinemann, Oxford, 1976).

[3] H. Goldstein, C. Poole and J. Safko, Classical Mechanics (Pearson Education, Essex, 2011), $3^{\mathrm{a}}$ ed.

[4] P.M. Dirac, The Principles of Quantum Mechanics (Clarendon Press, Gloucestershire, 1982), $4^{\mathrm{a}}$ ed.

[5] R.P. Feynman and A. Hibbs, Quantum Mechanics and Path Integrals (McGraw-Hill, New York, 1965).

[6] L. Landau and E. Lifshitz, The Classical Theory of Fields (Butterworth Heinemann, Oxford, 1975), $4^{\text {a }}$ ed.

[7] J.J. Sakurai, Advanced Quantum Mechanics (AddisonWesley Publishing Company, Reading, 1967).

[8] A.O. Barut, Electrodynamics and Classical Theory of Fields 83 Particles (Dover publications, New York, 1980). 